\title{
Kommentar zum Artikel «Recht auf Versicherungsleistungen» [1]
}

Rolf H. Adler

1 Meyer U. Krankheit als leistungsauslösender Begriff im Sozialversicherungsrecht. Schweiz Ärztezeitung. 2009;90(14):585-8.

Korrespondenz: Prof. Rolf H. Adler Leiserenweg 4 CH-3122 Kehrsatz Tel. 0319615496

rolf.adler@tele2.ch
Ulrich Meyer beleuchtet in seinem Beitrag, wie das Recht einen Rechtsanspruch auf Versicherungsleistung verweigert, wenn ein Mensch sich krank fühlt, krank ist, die Anspruchsgrundlage aber nicht bewiesen und nicht beweisbar ist. Diese Situation ist für den Arzt und auch für den Juristen unbefriedigend. Es gilt zu klären, warum ein Teil der so Kranken aus rechtlicher Sicht nicht als rentenwürdig betrachtet wird, obwohl der Betroffene sich offensichtlich so krank fühlt, dass er nicht zu arbeiten, am Familienleben nicht mehr teilzunehmen und keine Freizeitaktivitäten mehr auszuüben vermag.

Um diese unbefriedigende Situation zu klären und eine Lösung vorzuschlagen, wähle ich als Diskussionsbasis das Thema Evidenz und Evidenz-basierte Medizin. Dabei kürze ich, denn sonst müsste ich bei Descartes (1596-1650) beginnen und via Locke (16321704), Hume (1711-1776), J. von Uexküll (1864-1944), Piaget (1896-1980), Peirce (1836-1914) bis zu Th. von Uexküll (1908-2004) einen Bogen schlagen, um zu ar-
Lehm in ihre Tasche, das sie im Entree auf dem Boden entdeckt hat. (Es enthält den Abdruck eines Stiefelabsatzes, ein Indiz, das zum Mörder führen wird.)

Sie begibt sich später zur Polizeistation und wartet auf den Inspektor, der bald erscheint, die Hände hochwirft und ausruft: «Sie kommen doch nicht schon wieder, um mich auf einen Mord aufmerksam zu machen, diesmal um mir beizubringen, dass Mr. Enderby ermordet worden ist.» «Genau das habe ich vor», erwidert Miss Marple. Der Inspektor macht sich lustig über sie: «Sie wissen doch, dass Mr. Enderby herzkrank war, die Untersuchung der Leiche hat dies bestätigt.» Miss Marple beharrt auf ihrem Verdacht und bedeutet ihm hartnäckig, dass sie den Mord in diesem Falle selber aufklären wird.

Der Inspektor nimmt, wie die Juristen und die meisten begutachtenden Ärzte, die bekannte Herzerkrankung und den Autopsiebefund zur Kenntnis, und sie stellen die Diagnose «lang anhaltende Herzkrankheit mit akutem Herztod, am wahrscheinlichs-

\section{Jurist und Arzt werden den Kranken erst gerecht, wenn sie bio-psycho-sozial denken, den Menschen also als offenes und geschlossenes System betrachten}

gumentieren. Ein Detektivfilm mit Miss Marple hilft mir dabei. Er heisst «Murder at the Gallop» bzw. «Der Wachsblumenstrauss». Miss Marple und ihr Freund, der Bibliothekar, verkaufen für gute Zwecke Abzeichen in einem Städtchen. Sie gelangen ans Parktor des schwerreichen Mr. Enderby. Miss Marple stösst das schmiedeeiserne Tor auf. Beide gelangen über die Auffahrt ans Portal des schlossartigen Hauses. Auf wiederholtes Klingeln meldet sich niemand. Miss Marple forsch, ihr Begleiter zögernd treten durch die unverschlossene Tür ins grosszügige Entree ein, von dem eine breite Freitreppe auf die Galerie führt. Dort erscheint jetzt Mr. Enderby im Schlafrock, greift sich ans Herz, bricht zusammen und stürzt die Treppe hinunter. Er bleibt zu Füssen der beiden Besucher regungslos liegen. Miss Marple stellt seinen Tod fest. Um das Geschehen näher zu verstehen, steigt sie die Freitreppe hoch, öffnet die Tür, aus der Mr. Enderby getreten ist und schreckt zusammen, als ihr eine riesige, garstige Katze aus dem Dunkel des Zimmers heraus über die Schulter springt und verschwindet. Bevor die beiden das Haus verlassen, steckt Miss Marple noch ein Stück ten durch eine Rhythmusstörung». Sie verstehen den Menschen als ein «offenes» System, zugänglich ohne dass seine Geschichte, seine Persönlichkeit und seine Beziehungen zur Umgebung eine Rolle spielen. Sie denken gemäss einem mechanistischen, biomedizinischen Konzept. Miss Marple weiss vom bekannten Herzleiden des Mr. Enderby (bio-medizinischer Aspekt seines Leidens). Ihr ist aber ebenso vertraut, dass er an einer Katzenphobie leidet und nie eine Katze in seinem Anwesen geduldet hat (psychologischer Aspekt). Die Katze muss also heimlich in sein Haus gebracht worden sein. Sie kennt auch seine Familie. Er hat vier aufs Erbe versessene Neffen (sozialer Aspekt). Sie denkt also in einem bio-psycho-sozialen Konzept. Der BioAnteil repräsentiert den «offenen» Teil dieses Konstrukts. Hätte Mr. Enderby unten an der Treppe noch gelebt, hätte er von jedermann, anonym, der sich auf Reanimieren versteht, gemäss dem von Intensivstationsärzten entworfenen Algorithmus reanimiert werden können. Die psychischen und sozialen Elemente des Leidens von Mr. Enderby sind jedoch in seiner Psyche eingeschlossen wie das Uhrwerk in eine Uhr, 


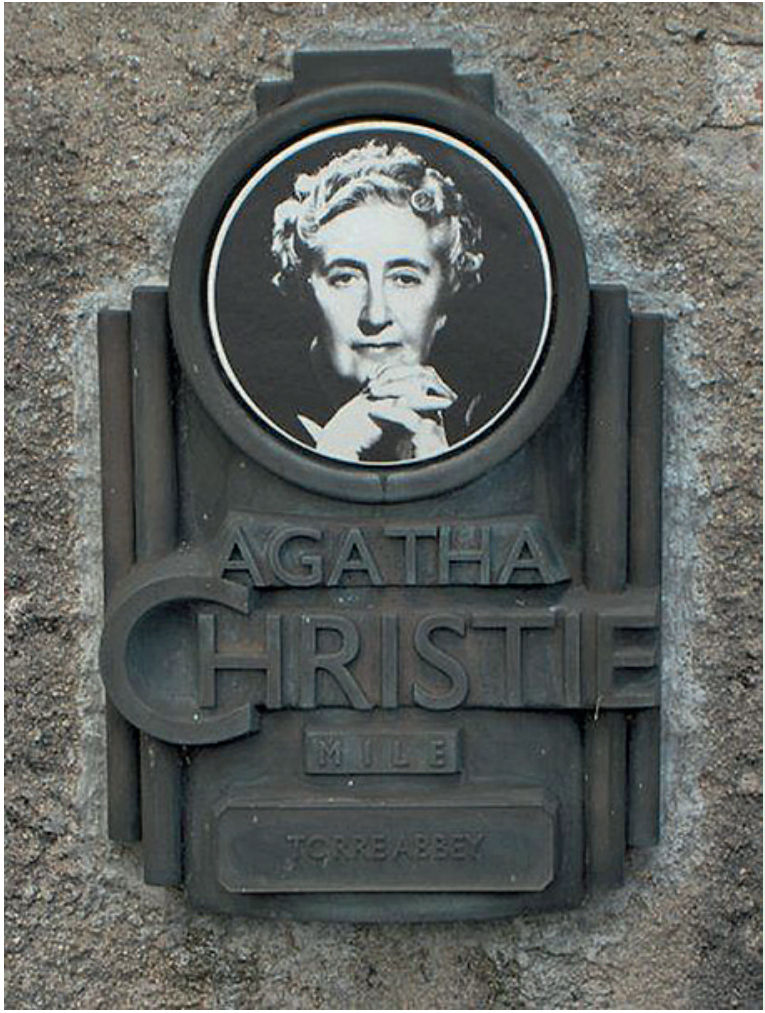

Alle Aspekte einer Tat berücksichtigen: so lautet das Motto von Agatha Christies Hobbydetektivin «Miss Marple». Immer interessiert sie sich auch für die psychologischen und sozialen Aspekte eines Falls. Wie ist das bei Ärzten und Juristen?

von dem der Beobachter lediglich die Zeiger und ihre Bewegungen wahrnehmen kann und Letztere auch nur, solange er noch lebt. (Der Pathologe sieht nur die stillstehenden Zeiger.) Deshalb wird das Psychische Affekte, Denken, Ideen, die individuelle Geschichte usw. - als «geschlossenes» System bezeichnet. Um die Uhr zu öffnen, zum Uhrwerk vorzudringen und seinen Mechanismus zu verstehen, benötigt der Arzt Empathie, um sich von Subjekt zu Subjekt verständigen zu können, eine Technik der Anamneseerhebung, die das geschlossene System «Patient» sachte zu öffnen vermag, und Kenntnisse der Entwicklungspsychologie, um das Uhrwerk deuten zu können.

Und hier komme ich zu Ulrich Meyer und zum Recht zurück. Die Wissenschaft vom Umgang mit offenen Systemen nennen wir eine Galilei'sche. Sie entspricht in der Medizin dem Bio-Konstrukt. Nach ihm gehen das Recht und die ärztlichen Gutachter vor und auch die sogenannte Evidenz-basierte Medizin. Das bio-psycho-soziale Konstrukt enthält den BioAnteil als Subsystem Das geschlossene System enthält auch eine Evidenz, die individuell-geschichtliche des Menschen.

Medizinische Forschung an Tier und Mensch weist eindeutig nach, dass psychosoziale Faktoren zum Erkranken, zur Krankheit und zum Verlauf in der Erkrankung beizutragen vermögen (wenn auch nicht in jedem Fall). So führt mentaler Stress - rechnen und dabei über eine mit Wut verbundene Erinnerung sprechen - bei Menschen mit erkrankten Koronararterien, die zum Erweitern der Kranzarterien ins Spital eintreten, zu einer erhöhten Verklumpung der an der Blutgerinnung beteiligten Blutplättchen.

\section{Medizinische Forschung weist nach, dass psychosoziale Faktoren zum Erkranken beitragen können}

\section{Folgerung}

Die Naturwissenschaft vom Menschen ist die Wissenschaft von der Natur des Menschen. Und diese ist unbestreitbar eine bio-psycho-soziale. Das schriftlich festgehaltene Recht entspricht in der Analogie zwischen Recht und Medizin dem heutigen Versicherungsrecht, dem sich Jurist und Arzt beugen müssen. Recht muss aber auch «billig» sein. Billig heisst in unserem $\mathrm{Zu}$ sammenhang «der Natur des Menschen angepasst», also sich der individuellen Geschichte des Einzelnen anschmiegend.

Jurist und Arzt werden den Kranken erst gerecht, wenn sie bio-psycho-sozial denken, den Menschen also als offenes und geschlossenes System betrachten. Das ist derzeit viel verlangt, aber unumgänglich. Erst dann werden Leiden verstanden und beweisbar. 